Bundesgesundheitsbl 2017 · 60:472-475

DOI 10.1007/s00103-017-2523-z

(C) Springer-Verlag Berlin Heidelberg 2017

\title{
Richtlinie der Gendiagnostik- Kommission (GEKO) für die Beurteilung genetischer Eigenschaften hinsichtlich ihrer Bedeutung für die Wirkung eines Arzneimittels bei einer Behandlung gemäß §23 Abs. 2 Nr. $1 \mathrm{~b}$ GenDG
}

\author{
In der Fassung vom 25.11.2016, veröffentlicht \\ und in Kraft getreten am 06.12.2016
}

\section{Vorwort}

Am 1. Februar 2010 ist in Deutschland das Gesetz über genetische Untersuchungen bei Menschen (Gendiagnostikgesetz - GenDG) in Kraft getreten. Die Aufgabe, Richtlinien im gesetzlichen Rahmen ( $\$ 23$ GenDG) für verschiedene Teilbereiche des GenDG zu erarbeiten, wurde der beim Robert Koch-Institut (RKI) eingerichteten Gendiagnostik-Kommission (GEKO) übertragen. Die GEKO ist aus 13 Sachverständigen aus den Fachrichtungen Medizin und Biologie, 2 Sachverständigen aus den Fachrichtungen Ethik und Recht sowie 3 Vertretern der für die Wahrnehmung der Interessen der Patientinnen und Patienten, der Verbraucherinnen und Verbraucher und der Selbsthilfe behinderter Menschen auf Bundesebene maßgeblichen Organisationen zusammengesetzt.

Die GEKO hat unter anderem den gesetzlichen Auftrag, in Bezug auf den allgemein anerkannten Stand der Wissenschaft und Technik eine Richtlinie für die Beurteilung genetischer Eigenschaften hinsichtlich ihrer Bedeutung für die Wirkung eines Arzneimittels bei einer Behandlung zu erstellen ( $\$ 23$ Abs. 2 Nr. 1b GenDG).
Die Beurteilung genetischer Eigenschaften hinsichtlich ihrer Bedeutung für Erkrankungen oder gesundheitliche Störungen sowie die Möglichkeiten, sie zu vermeiden, ihnen vorzubeugen oder sie zu behandeln sind in einer gesonderten Richtlinie der GEKO geregelt [1].

\section{Anwendungsbereich und Zweck der Richtlinie}

Die Richtlinie befasst sich auf der Basis des allgemein anerkannten Standes der Wissenschaft und Technik mit der Beurteilung hereditärer genetischer Eigenschaften geborener Menschen sowie von Embryonen und Föten während der Schwangerschaft in Hinblick auf die Wirkung eines Arzneimittels bei einer Behandlung.

Somatische genetische Veränderungen im Sinne nichthereditärer Eigenschaften sind vom Regelungsbereich des GenDG nicht erfasst und daher nicht Gegenstand dieser Richtlinie. Der in der Tumordiagnostik gebräuchliche Begriff „Companion Diagnostics“, der somatische und hereditäre genetische Varianten erfasst, wird in dieser Richtlinie zur Vermeidung von Missverständnissen nicht verwendet.
Die Richtlinie richtet sich an Ärztinnen und Ärzte, die eine genetische Untersuchung mit dem Zweck der Abschätzung der Wirksamkeit und Verträglichkeit der individuellen Arzneimitteltherapie durchführen. Diese schließt auch eine Ursachenabklärung bei Verdacht einer vorhandenen unerwünschten Arzneimittelwirkung oder nicht ausreichendem Ansprechen ein.

Diese Richtlinie dient nicht der Nutzenbewertung im Sinne einer leistungsrechtlichen Bewertung genetischer Diagnostik zum Zweck des Nachweises pharmakogenetischer Eigenschaften.

\section{Pharmakogenetische Untersuchungen im Sinne dieser Richtlinie}

Pharmakogenetik beschäftigt sich mit dem Einfluss genetischer Eigenschaften auf die Pharmakodynamik (Wirkqualität und -stärke), die Pharmakokinetik (Geschwindigkeit der Aufnahme, Metabolisierung und Ausscheidung) von Arzneistoffen sowie auf das Risiko von Überempfindlichkeitsreaktionen.

Der in dieser Richtlinie verwendete Begriff pharmakogenetische Untersuchung bezieht sich ausschließlich auf die 


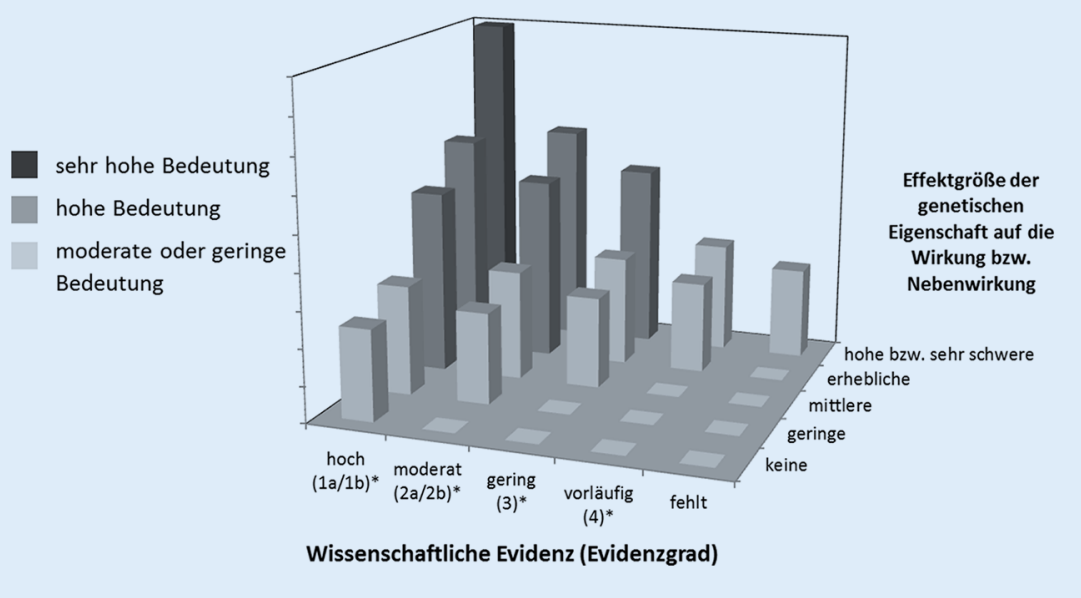

\ Schematische Darstellung für die Beurteilung einer genetischen Eigenschaft hinsichtlich ihrer Bedeutung bei der Anwendung von Arzneimitteln unter Berücksichtigung der vorhandenen wissenschaftlichen Evidenz für die genetische Assoziation (Gen-Arzneimittel-Interaktion) einerseits und dem Effekt dieser Eigenschaft bei Mutationsträgern andererseits. * PharmGKB „Levels of Evidence” [3]

Abklärung, ob genetische Eigenschaften im Sinne des $\$ 3 \mathrm{Nr}$. 4 GenDG vorliegen, die die Wirkung eines Arzneimittels beeinflussen können ( $\$ 3 \mathrm{Nr}$. 7c GenDG).

Diese nach GenDG als diagnostisch eingeordnete genetische Untersuchung mit dem Ziel, das Vorliegen einer genetischen Eigenschaft festzustellen, die die Wirkung eines Arzneimittels oder mehrerer Arzneimittel ganz oder teilweise beeinflussen kann, soll bei den betroffenen Personen zu einer Optimierung der Arzneimittelanwendung beitragen. Voraussetzung für die Durchführung einer pharmakogenetischen Untersuchung ist in jedem Fall, dass die betroffene Person nach $\$ 9$ GenDG und unter Berücksichtigung der entsprechenden Richtlinie der GEKO [2] aufgeklärt ist und nach $\$ 8$ Abs. 1 GenDG eingewilligt hat.

Vorgeburtliche pharmakogenetische Untersuchungen setzen voraus, dass eine Behandlung des Embryos oder Fötus mit einem Arzneimittel vorgesehen ist, dessen Wirkung durch bestimmte genetische Eigenschaften beeinflusst wird ( $\$ 15$ Abs. 1 GenDG).

Bei entsprechender Bedeutung der genetischen Eigenschaften können geeignete Algorithmen dazu beitragen, ggf. durch Dosisanpassung bzw. spezifische Auswahl von Arzneimitteln oder deren Vermeidung eine Verbesserung der Arzneimittelsicherheit und -wirksamkeit zu ermöglichen.

\section{Anforderungen}

Basis für eine pharmakogenetische Untersuchung ist die begründete Annahme, dass die entsprechende genetische Eigenschaft eine individuelle Bedeutung für die Anwendung oder Dosierung des klinisch indizierten Arzneimittels hat. Eine begründete Annahme setzt das Vorhandensein entsprechender wissenschaftlicher bzw. klinischer Evidenz der Assoziation der genetischen Eigenschaft zum Wirkungsgrad eines Arzneimittels oder dem Schweregrad einer unerwünschten Arzneimittelwirkung voraus.

\section{Bedeutung pharmakogenetischer Eigenschaften}

Für die betroffene Person können ihre pharmakogenetischen Eigenschaften folgenden Bedeutungsstufen zugeordnet werden:

1. sehr hohe Bedeutung

2. hohe Bedeutung

3. moderate oder geringe Bedeutung

Diese Beurteilung der Bedeutung pharmakogenetischer Eigenschaften basiert auf allgemeinen, nach dem derzeitigen und allgemein anerkannten Stand der Wissenschaft und Technik international konsentierten Erkenntnissen über ihren Einfluss auf die Wirkung (Wirksamkeit und Verträglichkeit) eines Arzneimittels und berücksichtigt auch die klinische Validität und den klinischen Nutzen ${ }^{1}$.

In Folge neuer wissenschaftlicher Erkenntnisse und Studien kann sich die Beurteilung pharmakogenetischer Eigenschaften hinsichtlich deren Bedeutung ändern. Die pharmakogenetischen Eigenschaften können an Bedeutung gewinnen und entsprechend zu einer höheren Einstufung führen; umgekehrt können diese in eine niedrigere Stufe fallen.

Die Bedeutungsstufe der pharmakogenetischen Eigenschaft ist relevant für die ärztliche Indikationsstellung einer genetischen Untersuchung im Rahmen einer medikamentösen Behandlung.

Im Folgenden werden die drei Stufen für die Beurteilung der Bedeutung pharmakogenetischer Eigenschaften auf Basis des derzeitigen Standes der Wissenschaft anhand von Beispielen erläutert:

\section{Beispiele von \\ pharmakogenetischen Eigenschaften mit sehr hoher Bedeutung}

Von sehr hoher Bedeutung und mit hoher klinischer Relevanz und Evidenz hinterlegt ist die Eigenschaft HLA-B`5701 für die Anwendung von Abacavir bei HIV ${ }^{2}$. Die Relevanz für die betroffene Person ergibt sich aus dem $48 \%$ igen Risiko einer lebensbedrohlichen Hautüberempfindlichkeitsreaktion (Steven-Johnson-Syndrom) [6].

Von sehr hoher Bedeutung sind bestimmte Mutationen des CFTR-Gens für die Therapie von Patienten mit zystischer Fibrose mittels Ivacaftor. Arzneimittel mit dem Wirkstoff Ivacaftor sind gemäß ihrer

\footnotetext{
1 "Klinischer Nutzen“ und "Klinische Validität" werden hier in Anlehnung an die international gebräuchlichen Begriffe "Clinical Utility" und "Clinical Validity" verwendet, die zu den Kriterien für die Beurteilung genetischer Eigenschaften bezüglich ihrer klinischen Bedeutung zählen (sog. "ACCE"-Schema [4]). Anforderungen an die analytische Validität genetischer Untersuchungen zu medizinischen Zwecken sind u.a. Bestandteil der Richtlinie nach §23 Abs.2 Nr.4 GenDG [5].

2 Der negativ prädiktive Wert der Assoziation der genetischen Eigenschaft mit der Hypersensitivität gegenüber Abacavir beträgt 100\% (Quelle: http://www.ema.europa.eu/docs/en_GB/document_library/Scientific_guideline/2016/05/ WC500205758.pdf, Zugegriffen: 20.11.2016)
} 
Zulassung nur bei Trägern bestimmter Mutationen indiziert. ${ }^{3}$ Hier ist die genetische Eigenschaft sowohl krankheitsrelevant als auch mit der Wirkung eines Arzneimittels assoziiert.

\section{Beispiele von \\ pharmakogenetischen \\ Eigenschaften mit hoher \\ Bedeutung}

Das Phase-II-Enzym Thiopurin-S-methyltransferase (TPMT) ist in die Metabolisierung z. B. von Azathioprin und Mercaptopurin involviert. Diese Purinanaloga haben diverse Nebenwirkungen. Homozygote Träger der nicht aktiven TPMT-Variante (Nichtmetabolisierer) entwickeln bei normaler Dosierung einer PurinanalogaTherapie regelhaft Leukopenien bis hin zur lebensbedrohlichen Panzytopenie. Die Messung der Enzymaktivität oder Genotypisierung der TPMT ermöglicht eine Anpassung der Dosis zur Vermeidung dieser Nebenwirkung. Jedoch können auch Arzneimittelwechselwirkungen eine TPMT-Hemmung bewirken. Durch die engmaschige Überwachung der Patienten mittels Blutbildkontrolle kann diese spezielle Nebenwirkung frühzeitig erkannt und somit die Sicherheit der Patienten verbessert werden. Die Europäische Arzneimittel-Agentur (EMA) weist darauf hin, dass vor Therapiebeginn eine Phäno- oder Genotypisierung geeignet sein kann, einen potenziellen TPMT-Mangel festzustellen. ${ }^{4}$

Weitere Beispiele betreffen die genetischen Eigenschaften der Cytochrom P450 (CYP) Isoenzyme, die zum Teil bei der interindividuell verschiedenartigen Metabolisierung trizyklischer Antidepressiva eine Rolle spielen. Hier kann der Zusammenhang zwischen Genotyp und Phänotyp weniger ausgeprägt sein als bei den zuvor genannten Beispielen. Der verminderte Abbau von Amitriptylin kann mit er-

\footnotetext{
3 Fachinformation der Europäischen Arzneimittel-Agentur (EMA): http://www.ema. europa.eu/docs/de_DE/document_library/ EPAR_-_Product_Information/human/002494/ WC500130696.pdf (Zugegriffen: 20.11.2016)

${ }^{4}$ Fachinformation der Europäischen Arzneimittel-Agentur (EMA): http://www.ema. europa.eu/docs/en_GB/document_library/ EPAR_-_Product_Information/human/002022/ WC500124645.pdf (Zugegriffen: 20.11.2016)
}

heblichen, ggf. sogar lebensbedrohlichen, Nebenwirkungen einhergehen. Umgekehrt sind CYP2D6-Gen-Duplikationen bekannt, die den Abbau von Amitriptylin beschleunigen und somit zu einem unzureichenden Ansprechen der Therapie führen können. Unter der Verabreichung des Arzneimittels kann der genetisch bedingte verminderte oder beschleunigte Abbau indirekt durch eine Plasmakonzentrationsbestimmung erkannt werden, die Feststellung einer genetischen Variante durch eine pharmakogenetische Untersuchung könnte das Risiko einer Über- bzw. Unterdosierung von vornherein vermeiden [7].

In seltenen Fällen kann eine genetische Eigenschaft individuell die Wirksamkeit eines Arzneimittels erheblich beeinflussen, ohne dass hierfür jedoch gesicherte Studien vorliegen. Ein Beispiel ist das homozygote Vorliegen eines prämaturen Stopp-Codons im CYP3A4-Gen, das z. B. dazu führen kann, dass das Immunsuppressivum Tacrolimus nur vermindert ausgeschieden werden kann. Bei nicht angepasster Dosis kann dieser Defekt zu erheblichen Nebenwirkungen ${ }^{5}$ führen.

\section{Beispiele für pharmakogenetische Eigenschaften mit moderater oder geringer Bedeutung}

Hierzu zählen beispielsweise genetische Eigenschaften, die Einfluss auf die Pharmakokinetik eines Arzneistoffes haben, ohne dass daraus bislang ein direkter klinischer Nutzen für die Behandlung abzuleiten wäre.

Valide Kenntnisse bestehen insbesondere im Hinblick auf die Beeinflussung der Plasmakonzentration von Arzneistoffen durch Enzyme des Arzneistoffwechsels. Beginnend mit der Beobachtung phänotypisch unterschiedlicher Ausscheidungsgeschwindigkeiten konnten durch den Fortschritt molekularbiologischer Methoden mit der Enzymaktivität assoziierte Genvarianten identifiziert werden. Eine hohe Korrelation vom Genotyp zur

\footnotetext{
${ }^{5}$ Der Begriff Nebenwirkung wird hier nach der gesetzlichen Definition in $\S 4$ Abs.13 des Arzneimittelgesetzes (AMG) als eine schädliche und unbeabsichtigte Reaktion auf ein Arzneimittel verwendet.
}

Enzymaktivität besteht insbesondere bei Varianten, die zu einem kompletten Aktivitätsverlust führen. Beispiele hierfür sind Varianten in den Phase-I-Enzymen Cytochrom P450 (CYP) 2D6 und CYP3A5. Durch diese Varianten wird die Pharmakokinetik von Arzneistoffen wie z.B. Metoprolol beeinflusst. Ein klinischer Nutzen der pharmakogenetischen Untersuchung z. B. für die Behandlung der Hypertonie oder Herzinsuffizienz ist allerdings nicht nachgewiesen. Die Feststellung dieser pharmakogenetischen Eigenschaften kann im Einzelfall zur Klärung unerwünschter Wirkungen beitragen.

In jüngerer Zeit mehren sich Hinweise, dass auch Varianten in Efflux- und insbesondere Aufnahmetransportern die Bioverfügbarkeit und in der Folge auch das Risiko unerwünschter Wirkungen von Arzneistoffen beeinflussen. So weisen homozygote Träger einer Variante im organischen Anionentransporter OATP1B1 (SLCO1B1) ein 17-fach erhöhtes Myopathierisiko unter Verabreichung vom $80 \mathrm{mg}$ Simvastatin auf. Die EMA hat dies zum Anlass genommen, in der Zulassung von Simvastatin-haltigen Arzneimitteln auf diese Assoziation und die Existenz von entsprechenden Genotypisierungstests hinzuweisen. ${ }^{6}$

\section{Begründung}

Die Bewertung genetischer Eigenschaften hinsichtlich ihrer Bedeutung für die Wirkung eines Arzneimittels bei einer Behandlung ist Aufgabe der biomedizinischen Wissenschaft. Im Gegensatz zur Identifizierung genetischer Ursachen für die Entstehung seltener (monogener Formen von) Erkrankungen ist die Feststellung des genetischen Anteils bei der interindividuellen Variabilität von Arzneimittelwirkungen oftmals schwierig bzw. es sind hierfür nur wenige geeignete Evaluierungsmöglichkeiten vorhanden. Die Effizienz von pharmakologischen Wirkstoffen kann teilweise sehr stark schwanken und ist meist abhängig von nicht-erbli-

\footnotetext{
${ }^{6}$ Page $6 / 19$, Guideline on key aspects for the use of pharmacogenomics in the pharmacovigilance of medicinal products EMA/CHMP/281371/2013: http://www.ema.europa.eu/docs/en_GB/document_library/Scientific_guideline/2015/11/ WC500196800.pdf (Zugegriffen: 20.11.2016)
} 
chen Faktoren (Alter, Ernährungszustand, Erkrankungen, Komedikation etc.). Für den genetischen Anteil bei unerwünschten Arzneimittelwirkungen gibt es derzeit kaum Schätzungen. Die Einschätzung der klinischen Validität und des klinischen Nutzens pharmakogenetischer Untersuchungen basiert auf der jeweils bestmöglichen medizinischen Evidenz. Diese kann aus sorgfältig konzipierten, randomisierten klinischen Studien gewonnen werden, es können unter Umständen jedoch auch Belege mit einem niedrigeren Evidenzlevel (z. B. aus Beobachtungsstudien) für eine Therapieentscheidung bzw. Indikationsstellung einer pharmakogenetische Untersuchung herangezogen werden [8]. Erste, auf einem wissenschaftlichen Konsens [9] beruhende Leitlinien zur Dosierungsänderung oder Auswahl von Arzneistoffen bei Vorliegen pharmakogenetischer Eigenschaften wurden vom Clinical Pharmacogenetics Implementation Consortium (CPIC) des US-amerikanischen PharmGKB Netzwerkes [10] und der Dutch Pharmacogenetics Working Group (DPWG) [11, 12] entwickelt. Die Europäische Arzneimittelbehörde (EMA) hat bereits heute etwa $15 \%$ ihrer bisher zugelassenen Arzneimittel mit Hinweisen auf spezifische, sowohl somatische als auch hereditäre pharmakogenetische Eigenschaften in den Produktinformationen ${ }^{7}$ versehen [13].

\section{Inkrafttreten}

Diese Richtlinie wird nach Verabschiedung ihrer endgültigen Form durch die GEKO mit der Veröffentlichung auf der Homepage des RKI wirksam.

7 Die Produktinformationen dienen als Grundlage für die sichere und gezielte Anwendung eines Arzneimittels.

\section{Literatur}

1. Gendiagnostik-Kommission beim Robert KochInstitut (2013) Richtlinie der Gendiagnostik-Kommission (GEKO) für die Beurteilung genetischer Eigenschaften hinsichtlich ihrer Bedeutung für Erkrankungen oder gesundheitliche Störungen sowie für die Möglichkeiten, sie zu vermeiden, ihnen vorzubeugen oder sie zu behandeln gemäß § 23 Abs. 2 Nr. 1 a GenDG. http://www.rki.de/DE/ Content/Kommissionen/GendiagnostikKommission/Richtlinien/Richtlinien_node.html.Zugegriffen: 20. Nov. 2016

2. Gendiagnostik-Kommission beim Robert Koch-Institut (2013) Richtlinie der Gendiagnostik-Kommission (GEKO) für die Anforderungen an die Inhalte der Aufklärung bei genetischen Untersuchungen zu medizinischen Zwecken gemäß § 23 Abs. 2 Nr. 3 GenDG. http://www.rki.de/DE/Content/Kommissionen/GendiagnostikKommission/Richtlinien/ Richtlinien_node.html. Zugegriffen: 20. Nov. 2016

3. Whirl-Carrillo M, McDonagh EM, Hebert JM et al (2012) Pharmacogenomics Knowledge for Personalized Medicine. Clin Pharmacol Ther 92:414-417

4. The Office of Public Health Genomics, Centers for Disease Control and Prevention (CDC) (2010) ACCE Model Process for Evaluating Genetic Tests. http://www.cdc.gov/genomics/gtesting/ACCE/ index.htm. Zugegriffen: 20. Nov. 2016

5. Gendiagnostik-Kommission beim Robert Koch-Institut (2013) Richtlinie der GendiagnostikKommission (GEKO) für die Anforderungen an die Qualitätssicherung genetischer Analysen zu medizinischen Zwecken gemäß § 23 Abs. 2 Nr. 4 GenDG. http://www.rki.de/DE/Content/Kommissionen/GendiagnostikKommission/Richtlinien/ Richtlinien_node.html. Zugegriffen: 20. Nov. 2016

6. Mallal S, Phillips E, Carosi G et al (2008) HLA$B * 5701$ screening for hypersensitivity to abacavir. N Engl J Med 358:568-579. doi:10.1056/NEJMoa0706135

7. Hicks JK, Swen JJ, Thorn CF et al (2013) Clinical Pharmacogenetics Implementation Consortium guideline for CYP2D6 and CYP2C19 genotypes and dosing of tricyclic antidepressants. Clin Pharmacol Ther 93:402-408

8. Rawlins M (2008) De testimonio: on the evidence for decisions about the use of therapeutic interventions. Lancet 372:2152-2161

9. Caudle KE, Klein TE, Hoffman JM et al (2014) Incorporation of pharmacogenomics into routine clinical practice: the Clinical Pharmacogenetics Implementation Consortium (CPIC) guideline development process. Curr Drug Metabol 15:209-217

10. Clinical Pharmacogenetics Implementation Consortium http://www.pharmgkb.org/page/cpic. Zugegriffen: 20. Nov. 2016
11. Swen JJ, Nijenhuis M, de Boer A et al (2011) Pharmacogenetics: from bench to byte - an update of guidelines. Clin Pharmacol Ther 89:662-673

12. Dutch Pharmacogenetics Working Group http:// www.pharmgkb.org/page/dpwg. Zugegriffen: 20. Nov. 2016

13. Ehmann F, Caneva L, Prasad K et al (2015) Pharmacogenomic information in drug labels: European Medicines Agency perspective. Pharmacogenomics J 15:201-210 